\title{
Materiales eco-compatibles reforzados a base de almidón de mandioca para aplicaciones agronómicas
}

\author{
Cassava starch-based reinforced \\ eco-compatible materials with \\ agronomic applications
}

Florencia Versino ${ }^{1,2}$, María A. García ${ }^{1,3}$

\footnotetext{
${ }^{1}$ Centro de Investigación y Desarrollo en Criotecnología de Alimentos (CIDCA), Universidad Nacional de La Plata (UNLP), Consejo Nacional de Investigaciones Científicas y Técnicas (CONICET) y Comisión de Investigaciones Científicas de la Provincia de Buenos Aires (CICPBA) CP:1900, La Plata, Buenos Aires, Argentina.

${ }^{2}$ Departamento de Ingeniería Química, Facultad de Ingeniería, UNLP, CP:1900, La Plata, Buenos Aires, Argentina.

${ }^{3}$ Departamento de Química, Facultad de Ciencias Exactas, UNLP, CP:1900, La Plata, Buenos Aires, Argentina. e-mail: florencia.versino@ing.unlp.edu.ar; magarcia@quimica.unlp.edu.ar
}

\section{RESUMEN}

La utilización de plásticos de cobertura o mulching en diversos cultivos puede aumentar los rendimientos, extender la temporada de crecimiento, controlar malezas, aumentar la eficiencia de los fertilizantes y mejorar las condiciones del suelo. Sin embargo, luego de su uso son fuente de contaminación ambiental y su remoción genera un costo adicional. Para este fin y otras aplicaciones de interés agronómico el uso de películas a base de almidón es una alternativa viable debido a su biodegradabilidad, procedencia de un recurso renovable y relativo bajo costo. En el presente trabajo se exponen los resultados obtenidos en el desarrollo y estudio de materiales biodegradables a base de almidón de mandioca reforzados con el bagazo de la raíz, evaluando su biodegradabilidad en suelo y el efecto de la exposición de las mismas a ciclos de luz UV-visible (foto-períodos) durante 60 días.

El agregado de fibra da lugar a películas más opacas y coloreadas, aumentó su permeabilidad al vapor de agua en un 53\%, e incrementó su resistencia máxima a la tracción y módulo elástico. Asimismo, durante el almacenamiento se observó que tanto las propiedades ópticas como la solubilidad de las películas se mantuvieron. No obstante los materiales evidenciaron signos de envejecimiento, ya que aumentó su rigidez y cristalinidad. Las películas sin fibra mostraron un aumento marcado del módulo elástico y la tensión máxima en la ruptura, así como una disminución de la elongación; mientras que en las películas reforzadas este efecto fue menor. Para evaluar la biodegradación, las muestras se enterraron en tierra fértil bajo condiciones ambientales controladas. Al cabo de 42 días de ensayo se observó que tanto los materiales reforzados como los controles presentaban una pérdida de peso mayor al 60\%, evidenciando su biodegradabilidad. En conclusión, es posible desarrollar materiales eco-compatibles reforzados de almidón de mandioca con posibles usos agronómicos.

Palabras clave: Almidón de mandioca, materiales biodegradables, cobertura, uso agronómico.

\section{ABSTRACT}

Plastic mulch is used in various crops production in order to increase yield, extend growing season, weed control, increase fertilizers efficiency and improve soil conditions. However, since such plastics removal and disposal represent not only an additional cost, but also a source of environmental pollution, interest in starch-based materials as a viable alternative for this and other agricultural applications has raised in the last years. In this regard, films based on starch present several advantages: biodegradability, renewable-resource based and relatively low cost. The present work exhibit the results obtained in the development and study of biodegradable 
materials based on cassava starch reinforced with cassava bagasse, evaluating their biodegradability in soil and the effects of their exposure to UV -visible light cycles (photo-periods) for 60 days. Fibre inclusion resulted in more opaque and coloured films, with water vapour permeability a 53\% higher, and increased maximum tensile strength and elastic modulus. During storage no changes in both optical properties and solubility of the films were observed. However, the materials showed signs of aging as its rigidity and crystallinity increased. Films without fibre addition showed a marked rise in the elastic modulus and maximum stress at break while their elongation at break decreased; such effects were less noticeable in reinforced films. In order to evaluate the materials biodegradability, samples were buried in fertile soil under controlled environmental conditions. After 42 days, it was observed that both reinforced materials and controls had a weight loss greater than $60 \%$, evidencing their biodegradability. In conclusion, it is possible to develop eco-compatible cassava starch reinforced materials with potential agronomic uses.

Keywords: Cassava starch, biodegradable materials, mulch, agronomic use.

\section{INTRODUCCIÓN}

La creciente preocupación por el impacto de los fertilizantes minerales, fungicidas y herbicidas sobre el medio ambiente y la salud humana requiere el desarrollo de técnicas agronómicas alternativas que puedan reducir el uso de estos productos. Entre estas estrategias, la solarización del suelo es un método utilizado para aumentar la temperatura del suelo mediante el uso de películas plásticas para retener la energía de la radiación solar, a la vez que resulta una técnica prometedora para controlar patógenos y malezas [1].

Se denomina mulching a la práctica agronómica de cobertura del suelo, generalmente con materiales plásticos, para la conservación del suelo y del agua y favorecer el crecimiento de las plantas [2]. Esta práctica ampliamente utilizada en frutihortícolas permite aumentar los rendimientos, anticipar la cosecha, minimizar la dependencia de herbicidas y pesticidas, proteger los productos alimenticios y conservar de forma más eficiente el agua disponible, contribuyendo así a una producción agrícola más sostenible. Una gran parte de los plásticos utilizados en la agricultura son las películas plásticas que se utilizan en invernaderos, como túneles sobre hileras de cultivos, cubiertas de ensilado y sobre todo como mulching. Las películas plásticas agrícolas convencionales utilizadas actualmente son polietileno de baja densidad (en algunos casos polietileno de alta densidad), policloruro de vinilo, polibutileno o copolímeros de etileno con acetato de vinilo. Un efecto secundario negativo grave asociado con el uso de estos materiales sintéticos es la eliminación de los desechos plásticos agrícolas producidos cada año. El reciclado de estos materiales que requiere mucho tiempo lo que conlleva a un elevado costo de mano de obra. En general, se dejan en el campo o se queman de forma no controlada liberando sustancias nocivas con las consecuencias negativas obvias asociadas al medio ambiente.

Una alternativa prometedora que contribuya a la solución a este problema es la utilización de películas biodegradables producidas con materias primas renovables y de bajo costo como el almidón [3]. En un trabajo previo se desarrollaron películas a base de almidón de mandioca reforzadas con las fibras remanentes del bagazo de la extracción de dicho almidón [4]. Se caracterizó la composición química y la distribución de tamaño de partículas del bagazo; se demostró que con $25 \%$ de glicerol como plastificante se obtenían materiales con óptimas propiedades mecánicas y de barrera que mantenían su capacidad de termosellado.

Los materiales biodegradables que se utilicen con fines agrícolas deberían cumplir con tres requisitos críticos de diseño: lograr un buen comportamiento mecánico, conservarlo durante su aplicación en condiciones de campo y lograr una biodegradación del $100 \%$ en el suelo preferentemente antes de la siguiente estación de cultivo [5]. Para ser parte de sistemas agrícolas sostenibles, la biodegradación in situ de los materiales de mulching debe ocurrir dentro de un plazo razonable (menor a 2 años según lo propuesto por la nueva norma ASTM D5988-12 de biodegradación en suelo) y la calidad del suelo no debe verse afectada negativamente [6]. Otro parámetro relevante en las aplicaciones agronómicas es la estimación de la radiación fotosintéticamente activa (PAR) transmitida por el material en el rango de longitudes de onda $400-700 \mathrm{~nm}$. Esta radiación se asocia a la necesaria para la fotosíntesis, que es el proceso básico para la producción de cultivos [7]. Como consecuencia, la cantidad de radiación PAR transmitida por el material de recubrimiento, tiene gran importancia en relación con el crecimiento del cultivo y contribuye a la elección del mismo. Asimismo, debido a la exposición prolongada a los agentes climáticos, principalmente a la radiación solar, las películas ya sean sintéticas o naturales-biodegradables presentan alteraciones en sus propiedades físicas y mecánicas las que limitan su vida útil. 
Los objetivos del presente trabajo son estudiar la biodegradabilidad en suelo y el efecto de la exposición de las mismas a ciclos de luz UV-visible (foto-períodos) durante 60 días de materiales eco-compatibles biodegradables a base de almidón de mandioca reforzados con el bagazo de la raíz tuberosa.

\section{MATERIALES Y MÉTODOS}

\subsection{Preparación del material}

Se prepararon suspensiones acuosas de almidón de mandioca (3\%p/p) con agregado de bagazo de mandioca $(0$, 0,75 y $1,5 \% \mathrm{p} / \mathrm{p}$ ) las cuales se gelatinizaron en un baño termostático a $90^{\circ} \mathrm{C}$ durante 20 minutos y se plastificaron con glicerol $(0,75 \% \mathrm{p} / \mathrm{p})$. Las mismas se vertieron en placas de acrílico, se secaron a $50^{\circ} \mathrm{C}$ y se almacenaron a $20^{\circ} \mathrm{C}$ y $60 \% \mathrm{HR}$. El almidón de mandioca empleado fue extraído mediante un método simple previamente optimizado [8], a partir de raíces tuberosas cultivadas en la Estación Experimental Agropecuaria perteneciente al INTA Montecarlo (Misiones, Argentina). El remanente de la extracción del almidón (bagazo) se secó a $50^{\circ} \mathrm{C}$, se procesó y tamizó con un tamiz de malla normalizada $(500 \mu \mathrm{m})$ para luego ser utilizado como relleno. La composición química del bagazo y su distribución de tamaño de partículas, así como sus efectos sobre las propiedades mecánicas, ópticas y de barrera al vapor de agua de las películas de almidón de mandioca fueron estudiados en un trabajo anterior [4].

\subsection{Almacenamiento}

Las películas se almacenaron bajo condiciones controladas de humedad y temperatura $\left(53 \% \mathrm{HR}\right.$ y $\left.24^{\circ} \mathrm{C}\right)$, expuestos a ciclos de luz y oscuridad de 16 y 8hs, respectivamente, con el fin de reproducir las condiciones de exposición normal a la luz solar (foto-período). El efecto del almacenamiento durante 60 días se evaluó mediante medidas de: color superficial, espectrofotometría UV-visible, espesor, solubilidad, ensayos de barrido de frecuencia y ensayos de tracción en un analizador dinámico mecánico (DMA) y difracción de rayos X (DRX).

\subsection{Espesor}

El espesor de las películas estudiadas se determinó usando un medidor electrónico de espesor DCN-900 (Check Line, EUA) para materiales no conductores sobre sustratos no ferrosos. El promedio de al menos diez mediciones en diferentes posiciones para cada muestra fue empleado para la normalización de las propiedades ópticas y mecánicas ensayadas que se describen a continuación.

\subsection{Propiedades ópticas}

Las medidas de color superficial fueron realizadas con un colorímetro Minolta (CR 400, Osaka, Japón), calibrado con estándar ( $Y=93,80 ; x=0,3133 ; y=0,3193)$. Se registraron las coordenadas $L^{*}, a^{*}$ y b* de la escala CIE y se

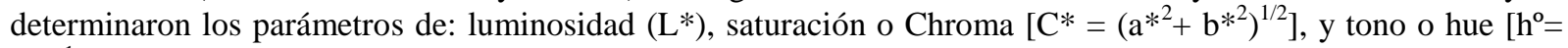
$\tan ^{-1}\left(\mathrm{~b}^{*} / \mathrm{a}^{*}\right)$ ] y la diferencia de color $(\Delta \mathrm{E})$.

El valor del coeficiente de transmisividad en el intervalo PAR y la capacidad de barrera a la luz UV de las películas se determinaron a partir de los espectros de absorbancia obtenidos en un espectrofotómetro U-1900 (HITACHI, Japón) entre 200 y $700 \mathrm{~nm}$. El valor de transmisividad en el intervalo PAR, es decir, la cantidad de radiación PAR transmitida por el material de recubrimiento se calculó como el valor promedio ponderado de la transmitancia $(\lambda)$ en el rango de 400 a $700 \mathrm{~nm}$ [9]. La barrera UV, por otro lado se calculó por integración del espectro entre 200 y 400nm, según lo descripto por CASTILLO et al. [10].

\subsection{Análisis termo-mecánico}

Las temperaturas e intensidades de los fenómenos de relajación de las películas, se determinaronmediante ensayos modulados de frecuencia en un analizador dinámico mecánico DMA Q800 (TA Instruments, EUA). Se realizaron barridos de múltiples frecuencias a una amplitud fija de -100 a $100^{\circ} \mathrm{C}$ a una velocidad de $5^{\circ} \mathrm{C} / \mathrm{min}$ 
sobre probetas de $30 \times 6 \mathrm{~mm}$. Las temperaturas de los procesos de relajación asociados a las temperaturas de transición vítrea ( $\mathrm{Tg}$ ), se midieron utilizando el software Universal Analysis 2000 a partir de las curvas de los módulos de almacenamiento (E') y pérdida (E') y las curvas tan $\delta$ (E'/E') en función de la temperatura [11].

\subsection{Difracción de rayos $X$}

Con el fin de estudiar las variaciones en el grado de cristalinidad de las muestras se realizaron ensayos de difracción de rayos X (DRX) en un difractómetro Philips PW 1390 (Holanda) empleando radiación $\mathrm{K} \alpha$ de $\mathrm{Cu}(\lambda$ $=1,5418 \AA$ ), $40 \mathrm{kV}$ de voltaje y $20 \mathrm{~mA}$ de corriente.

\subsection{Propiedades mecánicas}

El comportamiento mecánico de las películas se analizó mediante ensayos de esfuerzo controlado en un analizador dinámico mecánico DMA Q800 (TA Instruments, EUA) sobre probetas de las mismas dimensiones que para los ensayos de frecuencia. Como parámetros mecánicos de los materiales estudiados, se evaluaron la resistencia máxima a la tracción y el porcentaje de elongación antes de la ruptura, así como el módulo de elasticidad $\left(\mathrm{E}_{\mathrm{c}}\right)$, el cual fue determinado a partir de la pendiente inicial de la curva de esfuerzo-deformación.

\subsection{Solubilidad}

Se cortaron muestras de $2 \mathrm{~cm} \times 2 \mathrm{~cm}$ de cada película, se pesaron $(0,0001 \mathrm{~g})$ y se colocaron en vasos de ensayo con $80 \mathrm{ml}$ agua destilada. Las muestras se mantuvieron en condiciones constantes de agitación durante $1 \mathrm{~h} \mathrm{a} 20^{\circ} \mathrm{C}$. Las piezas restantes de las películas después de remojo se secaron en estufa a $105 \pm 1^{\circ} \mathrm{C}$ hasta peso constante. La solubilidad en película (\%) se calculó como sigue:

$\%$ Solubilidad $=\left[\frac{\text { peso } \text { seco } \text { inicial-peso seco } \text { final }}{\text { peso } \text { seco } \text { inicial }}\right] \times 100$

Todas las muestras se analizaron al menos por duplicado.

\subsection{Biodegradación}

Muestras de películas plastificadas con glicerol y distinto contenido de bagazo (0 y 1,5\%p/p) fueron cortadas en discos de $4 \mathrm{~cm}$ de diámetro, colocadas en un soporte constituido por una malla plástica, y enterradas en recipientes a una profundidad de $5 \mathrm{~cm}$ desde la superficie a fin de asegurar la degradación aeróbica. Como medio se utilizó la propia microflora presente en la tierra utilizada, la cual presentaba las siguientes características y composición: humedad: $35-40 \%$, cenizas: $40-45 \%$, relación $\mathrm{C} / \mathrm{N}: 7,7$, materia orgánica: $15-20 \%$, pH: 6,2 y conductividad eléctrica: $1,1 \mathrm{mS} . \mathrm{cm}^{-1}$. De acuerdo a la metodología descripta en la Norma ASTM D5988-03 se adicionó $1 \mathrm{~g}$ de compost cada $25 \mathrm{~g}$ de tierra. Las características fisicoquímicas y la composición del compost utilizado fueron: humedad: $40-45 \%$, cenizas: $25-30 \%$, relación C/N: 20-25, materia orgánica: $25-30 \%$, pH: 6,06,5 y conductividad eléctrica: $0,5-1,0 \mathrm{mS} . \mathrm{cm}^{-1}$.

Los recipientes se acondicionaron a una temperatura y una humedad relativa controlada de $24^{\circ} \mathrm{C} \mathrm{y}$ $53 \% \mathrm{HR}$, respectivamente, tomadas como condiciones ambientales medias. En todos los casos las películas se regaron frecuentemente con un rociador. Similares condiciones fueron utilizadas por LUDUEÑA et al. [12] evaluando la biodegradabilidad de envases compuestos por policaprolactona y fibras de lignocelulosa. Se realizaron medidas de la variación de peso para evaluar si las películas expuestas a un medio propicio para su biodegradación sufrían pérdidas de masa como consecuencia de dicho proceso. La variación de peso a los diferentes tiempos de ensayo se cuantificó gravimétricamente. La pérdida de peso (\%) se determinó una vez que las muestras fueron desenterradas y cuidadosamente limpiadas con un cepillo para no dañar su estructura y minimizar los errores asociados a cuantificar masa debida a la presencia de partículas ajenas a la matriz. Se evaluaron distintos tiempos de exposición $(0,7,14,21,28,35$ y 42 días) para hacer un seguimiento del grado de avance de la degradación de los materiales. En todos los casos se realizaron inspecciones visuales de las películas luego de haberlas extraído de los recipientes a los distintos tiempos de ensayo y se tomaron fotografías de las 
mismas. Para comparar la velocidad de degradación de los materiales estudiados, se determinó el tiempo medio necesario para la degradación del 50\% del material, mediante el ajuste de los resultados obtenidos a la ecuación de Boltzmann de tipo sigmoidea [13]:

$D_{t}=\frac{D_{0}-D_{\infty}}{1+e^{\left(\frac{t-t_{50}}{d t}\right)}}+D_{\infty}$

donde $D_{t}$ es el porcentaje de degradación evaluado por diferencia de peso con la condición inicial normalizada, $\mathrm{D}_{0}$ y $\mathrm{D}_{\infty}$ son los porcentajes de degradación iniciales y finales considerados como 0 y $100 \%$ respectivamente, $\mathrm{t}_{50}$ representa el tiempo necesario para la degradación del $50 \%$ de la muestra y dt es un parámetro empírico de ajuste que se relaciona con la velocidad de degradación.

\subsection{Análisis estadístico}

Para el análisis estadístico de resultados se utilizó el software Systat ${ }^{\circledR}$ (versión 10.0). Se efectuó el análisis de varianza (ANAVA) y la comparación de medias mediante la prueba de la diferencia mínima significativa (LSD) de Fisher, con un nivel de significación $\mathrm{p}=0,05$.

\section{RESULTADOS Y DISCUSION}

\subsection{Películas reforzadas}

El efecto del agregado de bagazo se ha descripto en un trabajo anterior [4]. Se observó que una disminución en la barrera al vapor de agua (WVP) con el agregado del material de refuerzo, siendo la WVP de los materiales reforzados con 1,5\% de bagazo hasta un 53\% mayor que las películas control con igual contenido de plastificante. Además, el agregado de fibra da lugar a materiales más opacos y coloreados con mayor capacidad de barrera UV. De forma análoga, se evidenció un incrementó en la resistencia máxima a la tracción y el módulo elástico de los materiales reforzados cuanto mayor fuese el contenido de fibra, sin embargo contenidos mayores a $1,5 \%$ de relleno dan lugar a películas más frágiles con baja elongación y tenacidad.

\subsection{Almacenamiento}

Pasados 60 días de almacenamiento bajo condiciones controladas de humedad y temperatura y foto-períodos cíclicos de luz, se evidenció un comportamiento distinto entre las películas con diferentes contenidos de fibra. Por un lado, como se ha descripto en un trabajo anterior [4], el agregado de fibra provoca grandes cambios en el aspecto y color de las películas, siendo estas más opacas, con valores de $\mathrm{L}, \mathrm{C}^{*} \mathrm{y} \mathrm{h}^{\circ}$, significativamente menores $(\mathrm{p}<0,05)$ los cuales contribuyen a una gran diferencia de color $(\Delta \mathrm{E})$. Por otro lado, el efecto del almacenamiento sobre el aspecto de las muestras también resultó significativo ( $\mathrm{p}<0,05)$ : las muestras control $(0 \%)$ presentaron una disminución del $35,6 \%$ en el $\Delta \mathrm{E}$ y un leve aumento $(2,2 \%)$ en la luminosidad (L) y una disminución del 14,3\% en la saturación o intensidad de color $\left(\mathrm{C}^{*}\right)$. El agregado de fibra atenuó dicho efecto, siendo la disminución en $\mathrm{C}^{*}$ menor cuanto mayor el contenido de fibra $(8,4 \%$ y $0,7 \%$ para las muestras con 0,75 y $1,5 \% \mathrm{p} / \mathrm{p}$ de fibra, respectivamente) y la misma no resultó significativa $(\mathrm{p}>0,05)$. No obstante, la variación en $\Delta \mathrm{E}$ resultó significativa $(\mathrm{p}<0,05)$ para las películas con mayor contenido de fibra que presentaron una importante disminución en el tono $\left(h^{\circ}\right)$, efecto que puede atribuirse a una decoloración de los componentes de la fibra sensibles a la radiación UV como la lignina. Los resultados obtenidos se exponen en la Tabla 1.

Además, las películas reforzadas presentaron una mayor opacidad y capacidad de barrera-UV que las muestras control, observándose una disminución general de la transparencia de los films con el almacenamiento, pero las variaciones no fueron significativas $(\mathrm{p}>0,05)$. Como ya se mencionó el estudio de la capacidad de barrera-UV de los materiales reforzados resulta de interés para su aplicación como cobertura de suelo o mulching.

El contenido de humedad de las películas también resultó significativamente distinto $(\mathrm{p}<0,05)$ luego del almacenamiento controlado: las películas de almidón sin fibra se hidrataron, mostrando una variación en la humedad de la película desde $18,8 \pm 0,02 \%$ a $21,9 \pm 1,4 \%$. Por el contrario, las películas reforzadas se deshidrataron durante el proceso de almacenamiento, siendo este efecto más importante para las películas con mayor concentración de fibra (una disminución de $5,2 \%$ y de $18,3 \%$ para 0,75 y $1,5 \%$ p/p de bagazo 
respectivamente).

Tabla 1: Variaciones luego de 60 días de almacenamiento en los parámetros de color de las películas de almidón plastificadas con distinta concentración de bagazo: $0,0,75$, y $1,5 \%$ p/p, respecto a los valores iniciales.

\begin{tabular}{cccccc}
\hline DíAS & FIBRA $\%$ & $\mathbf{L}$ & $\mathbf{C}^{*}$ & $\mathbf{H}^{\mathbf{0}}$ & $\Delta \mathbf{E}$ \\
\hline 0 & 0 & $93,65 \pm 0,73^{\mathrm{d}}$ & $3,63 \pm 0,05^{\mathrm{b}}$ & $89,70 \pm 0,11^{\mathrm{b}}$ & $3,80 \pm 0,57^{\mathrm{b}}$ \\
\hline 0 & 0,75 & $92,56 \pm 0,19^{\mathrm{b}, \mathrm{c}}$ & $5,97 \pm 0,10^{\mathrm{d}}$ & $91,25 \pm 0,03^{\mathrm{d}}$ & $6,00 \pm 0,21^{\mathrm{c}}$ \\
\hline 0 & 1,5 & $91,73 \pm 0,72^{\mathrm{a}, \mathrm{b}}$ & $8,05 \pm 0,08^{\mathrm{e}}$ & $91,59 \pm 0,22^{\mathrm{d}}$ & $8,10 \pm 0,52^{\mathrm{d}}$ \\
\hline 60 & 0 & $95,71 \pm 0,06^{\mathrm{e}}$ & $3,11 \pm 0,05^{\mathrm{a}}$ & $88,52 \pm 0,03^{\mathrm{a}}$ & $2,44 \pm 0,01^{\mathrm{a}}$ \\
\hline 60 & 0,75 & $92,89 \pm 0,04^{\mathrm{c}, \mathrm{d}}$ & $5,47 \pm 0,07^{\mathrm{c}}$ & $91,26 \pm 0,45^{\mathrm{d}}$ & $6,00 \pm 0,10^{\mathrm{c}}$ \\
\hline 60 & 1,5 & $91,10 \pm 0,15^{\mathrm{a}}$ & $7,99 \pm 0,18^{\mathrm{e}}$ & $90,57 \pm 0,08^{\mathrm{c}}$ & $9,03 \pm 0,22^{\mathrm{e}}$ \\
\hline
\end{tabular}

Se presentan los valores medios \pm la desviación standard. Letras diferentes dentro de una misma columna indican diferencias significativas $(\mathrm{p}<0,05)$.
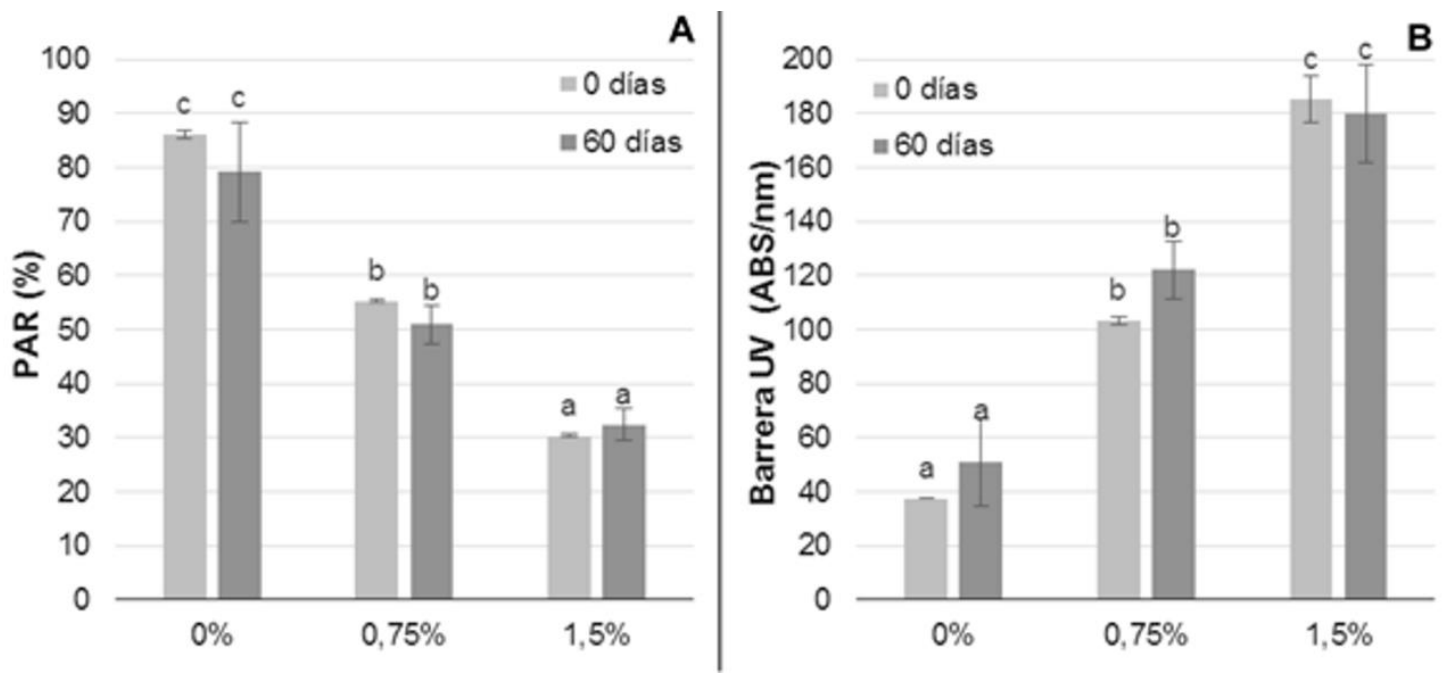

Figura 1: Comparación de transmisividad promedio en el intervalo PAR y capacidad de barrera UV obtenidos por espectrofotometría UV-visible para muestras con distinto contenido de fibra de bagazo de mandioca $(0,0,75$ y $1,5 \%$ p/p) a tiempo inicial y final.

Se conoce que las películas de almidón plastificadas con glicerol exhiben dos transiciones térmicas de segundo orden, una correspondiente a la fase rica en glicerol $\left(\mathrm{Tg}_{1}\right)$ y otra correspondiente a la fase rica en almidón $\left(\mathrm{Tg}_{2}\right)$ [14-16]. En general, el agregado de fibra solo modificó significativamente $(\mathrm{p}<0,05)$ la temperatura de transición vítrea de la fase rica en almidón, observándose un aumento de la misma con el contenido del relleno (Fig.3). Este comportamiento ha sido reportado en trabajos anteriores y por otros autores [14,15,17,18].

En general, luego del almacenamiento no se observaron diferencias significativas $(\mathrm{p}>0,05)$ en las temperaturas de transición vítrea de los materiales estudiados en función del tiempo independientemente de su composición (Tabla 2), con una excepción: las muestras con mayor contenido de fibra presentaron una disminución significativa $(\mathrm{p}<0,05)$ de la temperatura de transición vítrea correspondiente a la fase rica en plastificante $\left(\mathrm{Tg}_{1}\right)$. En los sistemas bifásicos la relación entre el contenido de humedad del material y sus temperaturas de transición vítrea no es sencilla, puesto que el agua total presente en el mismo podría interactuar diferencialmente y distribuirse en distinta proporción en cada una de las fases. No obstante, puede observarse una correlación entre los valores de humedad de las muestras y la temperatura de transición vítrea de la fase rica en almidón $\left(\mathrm{Tg}_{2}\right)$, la cual es mayoritaria en el sistema en estudio. 


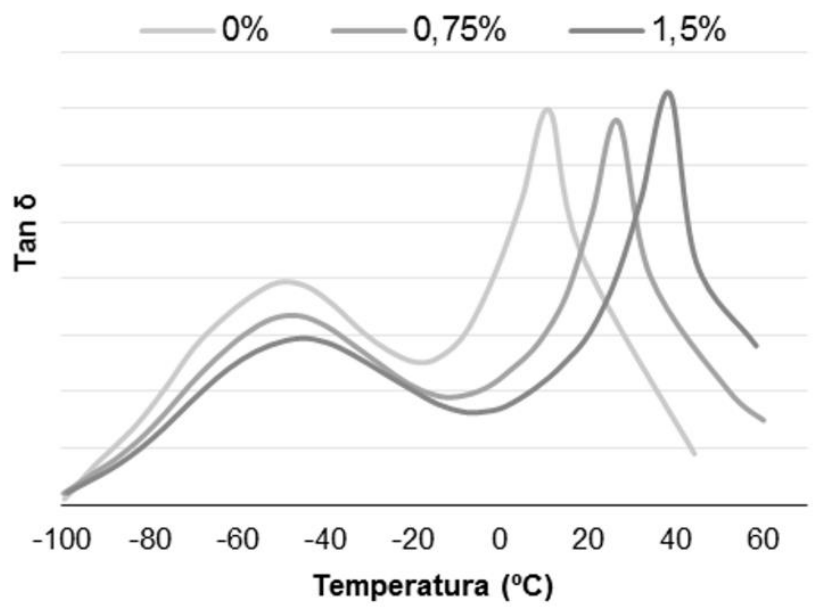

Figura 2: Barridos de frecuencia en función de la temperatura obtenidos por DMA para muestras con distinto contenido de fibra de bagazo de mandioca $(0,0,75$ y $1,5 \% \mathrm{p} / \mathrm{p})$ a tiempo inicial.

El estudio por difracción de rayos X (DRX) de las muestras control y con 1,5\% de fibra muestra que las películas de almidón de mandioca con y sin fibra presentan un grado de cristalinidad que no difiere significativamente $(\mathrm{p}>0,05)$. Sin embargo, luego del almacenamiento, las películas presentaron un aumento significativo $(\mathrm{p}<0,05)$ en la cristalinidad debido en ambos casos, como era de esperarse, a la retrogradación del almidón en la matriz (Tabla 2).

Tabla 2: Temperaturas de transición vítrea y grado de cristalinidad de muestras de films de almidón control ( $0 \%$ p/p) y con distinto contenido de bagazo de mandioca $(0,75$ y $1,5 \%$ p/p) a tiempo inicial y luego de 60 días de exposición a foto-períodos de luz bajo condiciones controladas de humedad y temperatura.

\begin{tabular}{ccccc}
\hline DÍAS & FIBRA (\%) & \multicolumn{2}{c}{ TEMPERATURA DE TRANSICIÓN VÍTREA } & GRADO DE CRITALINIDAD \\
\cline { 2 - 4 } & & $\left.\mathbf{T g}_{\mathbf{1}} \mathbf{(}^{\circ} \mathbf{C}\right)$ & $\left.\mathbf{T g}_{\mathbf{2}} \mathbf{(}^{\circ} \mathbf{C}\right)$ & GC (\%) \\
\hline 0 & 0 & $-49,83 \pm 0,18^{\mathrm{a}, \mathrm{b}}$ & $22,57 \pm 4,96^{\mathrm{a}, \mathrm{b}}$ & $3,60 \pm 0,79^{\mathrm{a}}$ \\
\hline 60 & 0 & $-47,04 \pm 2,72^{\mathrm{b}}$ & $16,02 \pm 8,48^{\mathrm{a}}$ & $5,38 \pm 0,62^{\mathrm{b}}$ \\
\hline 0 & 0,75 & $-44,81 \pm 0,37^{\mathrm{b}}$ & $33,12 \pm 3,84^{\mathrm{b}, \mathrm{c}}$ & $\mathrm{N} / \mathrm{D}$ \\
\hline 60 & 0,75 & $-48,95 \pm 2,05^{\mathrm{b}}$ & $44,38 \pm 1,02^{\mathrm{c}, \mathrm{d}}$ & $\mathrm{N} / \mathrm{D}$ \\
\hline 0 & 1,5 & $-46,07 \pm 1,69^{\mathrm{b}}$ & $51,50 \pm 6,31^{\mathrm{d}, \mathrm{e}}$ & $3,580 \pm 0,59^{\mathrm{a}}$ \\
\hline 60 & 1,5 & $-55,63 \pm 5,66^{\mathrm{a}}$ & $60,00 \pm 8,18^{\mathrm{e}}$ & $5,44 \pm 0,30^{\mathrm{b}}$ \\
\hline
\end{tabular}

Se presentan los valores medios \pm la desviación standard. Letras diferentes dentro de una misma columna indican diferencias significativas $(\mathrm{p}<0,05)$.

Por lo general, las películas que incluían el bagazo, presentaron una mayor resistencia mecánica que las películas de almidón de mandioca control evidenciada por una mayor fuerza máxima a la tracción y módulo elástico, y menor elongación a la ruptura. La misma tendencia se observó en las muestras almacenadas (Figura 3).

Contrariamente a lo esperado considerando la disminución de la $\mathrm{Tg}_{2}$ de la fase rica en almidón y el aumento en la humedad de las muestras: el control presenta una disminución en la elongación a la ruptura de aproximadamente un $50 \%$, efecto significativamente mayor $(\mathrm{p}<0,05)$ que para las películas reforzadas con bagazo. La disminución de la resistencia máxima a la tracción de las películas reforzadas 1,5\%, puede atribuirse a que una vez envejecidas las muestras, el almidón retrogradado tiene una interacción más débil con las partículas de bagazo, dando lugar a zonas de defecto estructural que disminuyen la fuerza del material. En concordancia con 
el aumento en la cristalinidad del material. En las muestras control, por otro lado, el aumento de la cristalinidad fomenta una mayor rigidez dando lugar a una mayor fuerza de ruptura y menor elongación. Las muestras que incluían $0,75 \%$ de fibra en su formulación presentaron un comportamiento intermedio, que puede deberse a la contribución de los efectos previamente analizados.
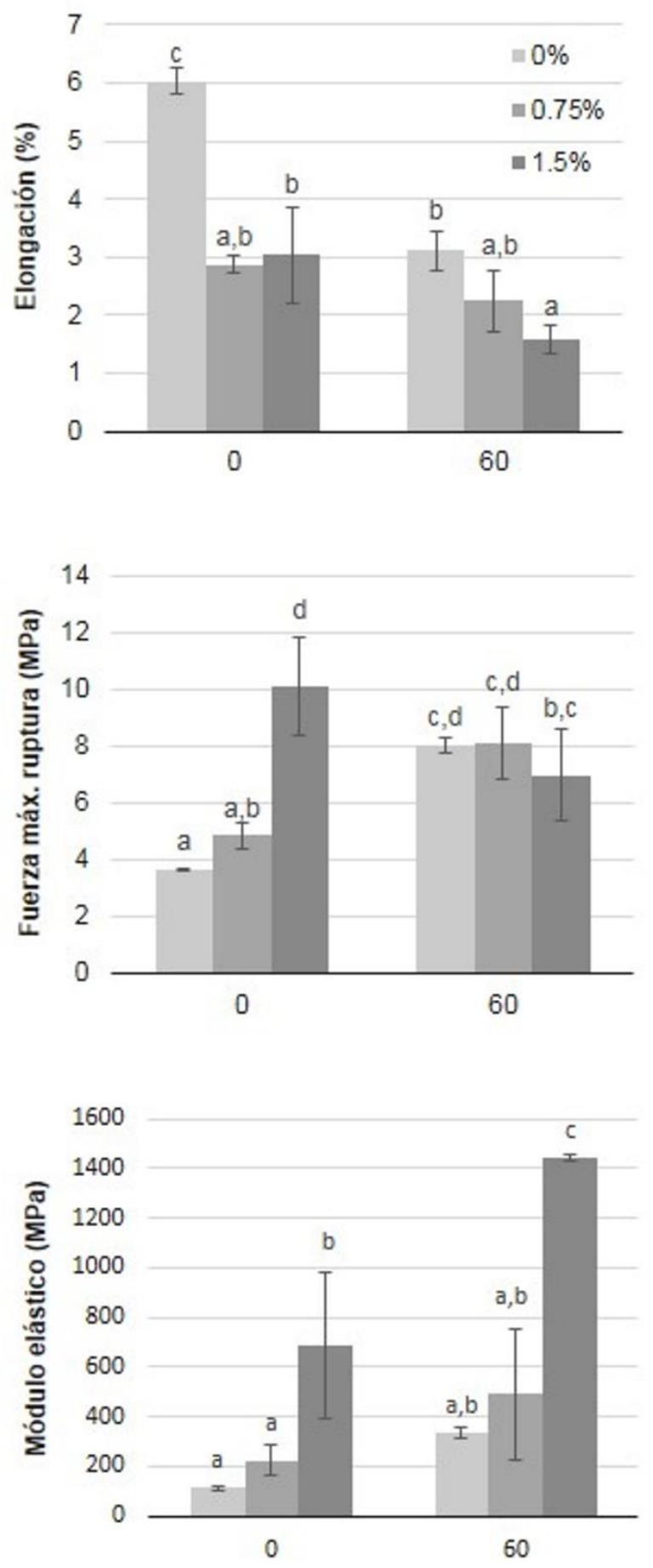

Figura 3: Propiedades mecánicas de películas de almidón plastificadas con distintas concentraciones de bagazo: 0\%; 0,75\%; y $1,5 \%$ a tiempo inicial (0 días) y final (60 días). 
Dicha disminución en la fuerza de interacción entre las partículas de relleno y la matriz se evidencia en el aumento de la solubilidad de las películas reforzadas con el tiempo de almacenamiento: la solubilidad aumenta de $9,40 \pm 0,61 \%$ a $17,15 \pm 0,95 \%$ para las películas reforzadas con $0,75 \%$ de bagazo, y $15,34 \pm 0,66 \%$ a $17,23 \pm 0,34 \%$. Sin embargo, este aumento fue solo significativo $(p<0,05)$ en el caso de las películas reforzadas con $0,75 \%$ de bagazo, posiblemente debido a una mayor contribución del efecto de fragilización de la matriz de almidón con el tiempo como en las matrices sin fibra. En forma análoga, la solubilidad de las muestras sin fibra inicialmente de $9,21 \pm 0,76 \%$ aumentó aproximadamente un $63 \%$, el cual se correlaciona con una evidente fragilización de la matriz de almidón mencionada anteriormente sumada a la absorción de agua por parte de la película.

Los resultados obtenidos indican que los materiales desarrollados sufren el envejecimiento por exposición a la radiación UV-visible, siendo este efecto menor en las películas reforzadas. Por tanto estos materiales serían adecuados para cultivos de ciclo corto, por ejemplo hortofrutícolas como la frutilla.

\subsection{Biodegradación}

En la Figura 4 se muestran los datos de biodegradación en tierra de las películas de almidón de mandioca control y reforzadas con 1,5\% de fibra de bagazo. Con el objetivo de ejemplificar el grado de degradación de las muestras, en la misma figura se incluyen fotografías de los materiales desenterrados dentro de la malla plástica luego de 15 y 42 días de compostaje.
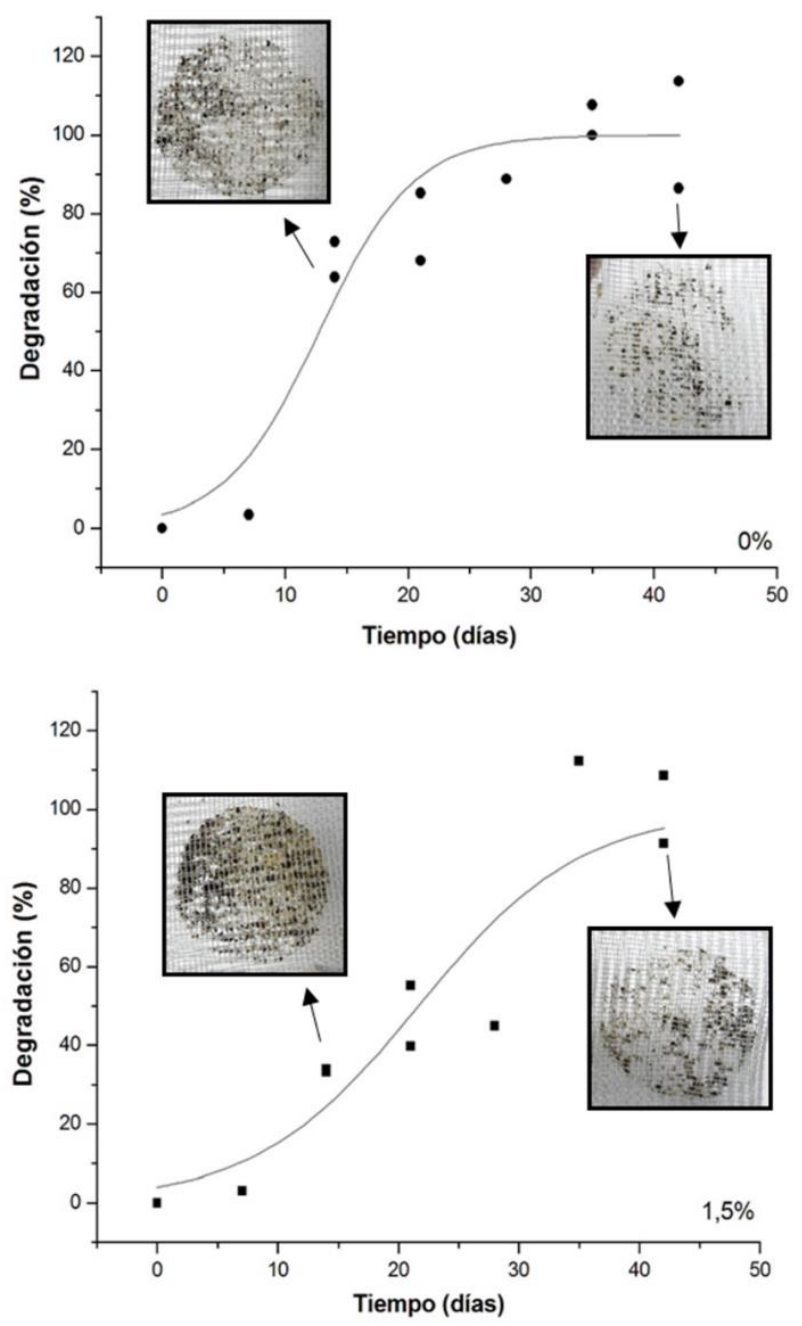

Figura 4: Biodegradación en tierra de muestras control $(\bullet)$ y con reforzada con 1,5\% de bagazo ( $\bullet$ ) en función del tiempo y curva de ajuste al modelo de Boltzmann. Las fotografías correspondes a las muestras en las mallas de plástico a los 15 y 42 días de exposición. 
El ajuste de los datos al modelo de Boltzmann resultó aceptable con un $\mathrm{r}^{2}$ de 0,9219 y 0,8882, aunque existe mayor dispersión a tiempos mayores. Los resultados indicaron que las películas reforzadas presentan un tiempo medio de degradación $t_{50}$ mucho mayor que el de las películas control, siendo $21,66 \pm 1,95$ y 12,75 $\pm 1,98$ respectivamente. El parámetro empírico de ajuste $d t$ fue de $6,79 \pm 1,90$ y $3,83 \pm 1,15$ para las películas reforzadas con $1,5 \%$ de fibra y las control respectivamente. Dicho parámetro se relaciona aunque de forma indirecta con la velocidad de degradación del material.

\section{CONCLUSIONES}

Las películas reforzadas presentaron una mayor opacidad y capacidad de barrera-UV que las control, observándose durante el almacenamiento en ambos casos una ligera disminución de la transparencia. Las películas biodegradables de almidón de mandioca sin agregado de fibra mostraron un aumento marcado del módulo elástico y la tensión máxima en la ruptura, así como una disminución de la elongación al cabo de 60 días de exposición a ciclos de luz UV-visible. Asimismo, en las películas reforzadas con el bagazo de la raíz este efecto fue menor. El aumento observado en la rigidez de los materiales desarrollados durante el almacenamiento se correlacionó con los cambios observados en la cristalinidad. Los resultados obtenidos indican que los materiales desarrollados sufren envejecimiento en las condiciones antes mencionadas, siendo este efecto menor en las películas reforzadas. Además, en todos los casos se demostró la biodegradabilidad de los materiales desarrollados, requiriendo las películas reforzadas con 1,5\% de fibra 43 días para su completa biodegradación y 25,5 días las películas control. Por tanto podría decirse que estos materiales serían adecuados para cobertura de suelos en cultivos de ciclo corto, por ejemplo frutihortícolas como la producción de frutillas.

\section{AGRADECIMIENTOS}

Este trabajo fue realizado con la ayuda de los proyectos: PICT 2011-1213 y 2015-0921 (ANPCyT, Argentina).

\section{BIBLIOGRAFÍA}

[1] BONANOMI, G., CHIURAZZI, M., CAPORASO, S., et al., "Soil solarization with biodegradable materials and its impact on soil microbial communities", Soil Biology and Biochemistry, v.40, n.8, pp. 1989-1998, 2008.

[2] PROSDOCIMI, M., TAROLLI, P., CERDÀ, A., "Mulching practices for reducing soil water erosion: A review", Earth-Science Reviews, v.161, pp. 191-203, 2016.

[3] SCARASCIA-MUGNOZZA, G., SCHETTINI, E., VOX, G., et al., "Mechanical properties decay and morphological behaviour of biodegradable films for agricultural mulching in real scale experiment", Polymer Degradation and Stability, v.91, n.11, pp. 2801-2808, 2006.

[4] VERSINO, F., GARCÍA, M. A., "Cassava (manihot esculenta) starch films reinforced with natural fibrous filler", Industrial Crops and Products, v.58, pp. 305-314, 2014.

[5] BRIASSOULIS, D., "Analysis of the mechanical and degradation performances of optimised agricultural biodegradable films", Polymer Degradation and Stability, v.92, n.6, pp. 1115-1132, 2007.

[6] LI, C., MOORE-KUCERA, J., LEE, J., et al., "Effects of biodegradable mulch on soil quality", Applied Soil Ecology, v.79, pp. 59-69, 2014.

[7] MONTEITH, J. L., UNSWORTH, M. H., Principles of environmental physics: Plants, animals, and the atmosphere, 4th(ed.), Oxford, UK, Elsevier Ltd., 2013.

[8] LÓPEZ, O. V., VIÑA, S. Z., PACHAS, A. N. A., et al., "Composition and food properties of pachyrhizus ahiparoots and starch", International Journal of Food Science \& Technology, v.45, n.2, pp. 223-233, 2010.

[9] SCARASCIA-MUGNOZZA, G., SCHETTINI, E., VOX, G., "Effects of solar radiation on the radiometric properties of biodegradable films for agricultural applications", Biosystems Engineering, v.87, n.4, pp. 479-487, 2004.

[10] CASTILLO, L., LOPEZ, O., LOPEZ, C., et al., "Thermoplastic starch films reinforced with talc nanoparticles", Carbohydrate Polymers, v.95, n.2, pp. 664-74, 2013.

[11] NINAGO, M. D., LOPEZ, O. V., LENCINA, M. M., et al., "Enhancement of thermoplastic starch final properties by blending with poly(varepsilon-caprolactone)", Carbohydr Polym, v.134, pp. 205-12, 2015. 
[12] LUDUEÑA, L., VÁZQUEZ, A., ALVAREZ, V., "Effect of lignocellulosic filler type and content on the behavior of polycaprolactone based eco-composites for packaging applications", Carbohydrate Polymers, v.87, n.1, pp. 411-421, 2012.

[13] ARRIETA, M. P., FORTUNATI, E., DOMINICI, F., et al., "Pla-phb/cellulose based films: Mechanical, barrier and disintegration properties", Polymer Degradation and Stability, v.107, pp. 139-149, 2014.

[14] GIRONÈS, J., LÓPEZ, J. P., MUTJÉ, P., et al., "Natural fiber-reinforced thermoplastic starch composites obtained by melt processing", Composites Science and Technology, v.72, n.7, pp. 858-863, 2012.

[15] MÜLLER, P., RENNER, K., MOCZO, J., et al., "Thermoplastic starch/wood composites: Interfacial interactions and functional properties", Carbohydrate Polymers, v.102, pp.821-829, 2014.

[16] NGUYEN VU, H. P., LUMDUBWONG, N., "Starch behaviors and mechanical properties of starch blend films with different plasticizers", Carbohydr Polym, v.154, pp. 112-120, 2016.

[17] LOPEZ, O. V., VERSINO, F., VILLAR, M. A., et al., "Agro-industrial residue from starch extraction of pachyrhizus ahipa as filler of thermoplastic corn starch films", Carbohydr Polym, v.134, pp. 324-332, 2015.

[18] MONTERO, B., RICO, M., RODRÍGUEZ-LLAMAZARES, S., BARRAL, L., et al., "Effect of nanocellulose as a filler on biodegradable thermoplastic starch films from tuber, cereal and legume", Carbohydrate Polymers, v.157, pp.1094-1104, 2016. 